\title{
73. ENERGY DISTRIBUTION IN GLOBULAR STAR CLUSTERS
}

\author{
L. H. ALLeR \\ University of California, Los Angeles \\ D. J. Faulkner \\ Mount Stromlo Observatory
}

The present investigation is concerned with the energy distributions in globular clusters. In a sense, energy-distribution measurements are comparable with multicolour photometry. The chief advantage is that narrower band-passes may be used and the entire spectrum traced, whereas in multi-colour photometry one is limited to effective wavelengths determined by the filter and a rather broad band-pass. On the other hand, broad band-pass photometry often permits one to cover a broader spectral range, and to work much faster. Since the time required for a single observation is much shorter, the observer is less at the mercy of the sky transparency. Hence a greater accuracy can be obtained.

We want to emphasize that energy distribution measurements such as those we have secured with purities of $24 \AA, 44 \AA$, or sometimes worse should be supplemented by slit spectrograms. A judicious combination of spectrograms of moderate dispersion and energy scans should provide valuable boundary conditions for testing hypotheses of the compositions of stars contained in star clusters.

Ideally, one could proceed as follows. From colour-magnitude arrays we synthesize the spectrum and energy distribution in a star cluster. For each interval of magnitude we know the number of stars and the distribution of these stars with respect to colour. The only adjustable parameter is the chemical composition. In our own Galaxy we can find stars of different chemical composition and can determine their energy distributions, colours, and spectral characteristics. For each assumed ratio of metals to hydrogen, one can construct what the integrated spectrum and energy distribution of a star cluster would look like-given its colour-magnitude array and luminosity function.

Thus, the problem is a well-determined one. The metal/hydrogen ratio in a cluster can be found provided we know:

(1) The colour-magnitude array for all stars contributing to the integrated light of the cluster;

(2) the luminosity function for all stars contributing appreciably to the integrated light of the cluster;

(3) the spectral class and the intensities of strategic luminosity-dependent and composition-dependent lines;

(4) the energy distribution in the integrated spectrum or the energy flux from the cluster at certain preselected wavelengths, with a band-pass $\Delta \lambda$;

(5) the energy distributions in stars of different temperatures, intrinsic luminosities, and chemical compositions. 
Unfortunately, this program cannot yet be carried out for a single cluster. For only a few clusters do we have detailed colour-magnitude arrays and luminosity functions. The spectroscopic observations have almost all been carried out with low dispersions and give only fragmentary data on line ratios needed for luminosity classifications and chemical compositions.

On the other hand, energy distributions have been measured for only a limited number of stars, most of which are of normal chemical composition. Data are available for only a few stars of abnormal chemical composition!

In the present study we have attempted to supply data on the energy distributions in several southern globular clusters. We have included several objects in the Large Magellanic Cloud, and one object, IC 419, in the Small Magellanic Cloud.

\section{Measurements of the Energy Distributions in Southern Globular Clusters}

In order to observe the spectral energy distributions in globular clusters, we employed the photoelectric spectrophotometer built at the University of Michigan by W. Liller with aid of a grant from the Rackham Foundation. The device was built originally to study particularly gaseous nebulae but has also been used for many other problems-stellar energy distributions, comets, etc. Since the instrument has been described in detail by Liller (1957) we restrict ourselves here to a brief description of its principles and operation.

Since the instrument was originally designed for an $f / 5$ system and has to be used in Australia on telescopes of such aperture ratios as $f / 12$ or $f / 18$, it was necessary to install an auxiliary optical system in front of the entrance slit-more properly called an entrance slot. We also provided a guiding diagonal consisting of an unsilvered, thick glass plate which was placed in the beam.

The entrance slot can be varied depending on the purity of the spectrum one wishes to obtain for an extended object. Slot A gives a purity of $6 \AA$, B one of $15 \AA$, $\mathrm{C}$ one of $25 \AA$, and $\mathrm{D}$ one of $59 \AA$. The length of the slot can be limited by a circular aperture which can be varied from 0.5 to $4.0 \mathrm{~mm}$ (corresponding to 11 to $88^{\prime \prime}$ at the focus of a 74-inch reflector). The light then falls on the Newtonian flat and passes to the collimator mirror and thence to the grating, which is rotated by a motor. With a 60 -cycle current the rate of scan is $30 \AA / \mathrm{min}$ at slow speed, $90 \AA / \mathrm{min}$ at medium speed, and $270 \AA / \mathrm{min}$ at high speed.

The spectrum formed by the grating is reflected by a second Newtonian flat through the exit slots and a Fabry lens, and thence to the photocell. The exit slots have purities of $2,9,19$, and $47 \AA$. Thus a combination of two B-slots (B-B) gives a purity of $24 \AA$, C-C gives a purity of $44 \AA$, and D-D gives a purity of $106 \AA-$ provided the cluster under observation fills the entrance slot.

Let $h_{\lambda}$ denote the deflection on the recording paper as normalized to some selected amplification. Let the true flux from a star or cluster in the wavelength range $\lambda$ to $\lambda+\mathrm{d} \lambda$ at the top of the Earth's atmosphere be denoted by $F \lambda$. Let $S_{\lambda}$ denote the combined effects of the sensitivity of the photocell and the transmission of the optics of the scanner plus that of the telescope. Then

$$
h_{\lambda}=S_{\lambda} F_{\lambda} \mathrm{e}^{-k_{\lambda} \sec z}
$$

where $k_{\lambda}$ is the coefficient of atmospheric extinction, and $z$ is the zenith distance. 
In actual practice we used $F(1 / \lambda) \cong F(\lambda) .\left(\lambda_{0} / \lambda\right)^{2}$ to compare energy distributions in wave number units. Hence $S(\lambda)$ is replaced by $S(\lambda)^{*}=S(\lambda) \cdot\left(\lambda_{0} / \lambda\right)^{2}$.

$A$ priori, the atmospheric extinction is not known; neither is the sensitivity function, $S_{\lambda}$. We determine these quantities in a straightforward way by observing a star whose energy distribution is known, and if necessary by taking another star which can be observed in the course of the night at widely different altitudes. In the northern hemisphere, where fundamental energy distributions have been carried out for a number of stars, one could often choose a single star to serve both purposes. In the southern hemisphere, however, it was necessary to pick a star near the celestial equator whose energy distribution had been measured; we used these stars to determine secondary standards of energy distribution for use in the southern hemisphere. This problem is being treated in a separate investigation. Actually, it turned out that we determined the atmospheric extinction each night by observing one of a relatively small number of southern stars that were to serve eventually as southern standards. These stars were in turn compared with northern standard stars. Hence it was possible to determine both $S_{\lambda}$ and the atmospheric extinction.

We observed the clusters as close to the meridian as possible so as to minimize effects of atmospheric extinction. Table 1 summarizes the individual observations. The first column gives the designation of the cluster and its position. The second gives the date of observation; all observations were secured between August 23, 1960, and March 18, 1961, so the year is not entered. The third column gives the instrument employed. "Bin" denotes the 26-inch reflector at the Mount Stromlo field station on Mount Bingar, near Yenda, N.S.W. The 50-inch reflector at Mount Stromlo was originally the "Great Melbourne Telescope" installed at the now-defunct Melbourne Observatory a century ago. It was purchased by Sir Richard Woolley and mounted on Mount Stromlo. The 74-inch reflector on Mount Stromlo was made by GrubbParsons. Column 4 gives the scan speed-fast, medium, or slow. With the 50-cycle current, the slow speed was $25 \AA / \mathrm{mm}$, the medium was $75 \AA / \mathrm{mm}$, and the fast was 225 $\AA / \mathrm{mm}$. The slots and apertures are denoted in the fifth column. For example, C4-C4 means entrance slot $\mathrm{C}$, aperture $4(3 \cdot 0-\mathrm{mm}$ diameter) and exit slot $\mathrm{C}$, aperture $4(5 \cdot 0$ $\mathrm{mm}$ diameter). Entrance aperture 5 has a diameter of $4.0 \mathrm{~mm}$; exit aperture 5 has a 5-mm diameter with a yellow filter. The spectral range covered is in the last column.

Figure 1 shows a scan of the spectrum of 47 Tucanae, as obtained with a medium speed on August 25 with the 50 -inch reflector. The spectral resolution is $24 \AA$. The yellow filter is introduced to cut out overlapping orders. Tracings were made both in the direction of increasing wavelength and in the reverse direction in order to secure a check on the guiding and the atmospheric transparency. These tracings were then superposed to obtain a final "mean" trace. The discrepancies are larger than we would like-probably they are at least partly due to the drifting of individual stars out of and into the slot. For this reason it is advantageous to use a small telescope in working on a large extended cluster such as $\omega$ Centauri:

Figure 2 shows a scan of NGC 1866, as observed with the 74-inch reflector on Mount Stromlo. Notice the prominent hydrogen lines, characteristic of a spectrum near A5, and the atmospheric line at 5577, which serves as a valuable fiducial mark. Here the spectral resolution is $44 \AA$. 
TABLE 1

SOUTHERN HEMISPHERE GLOBULAR CLUSTER SCANS

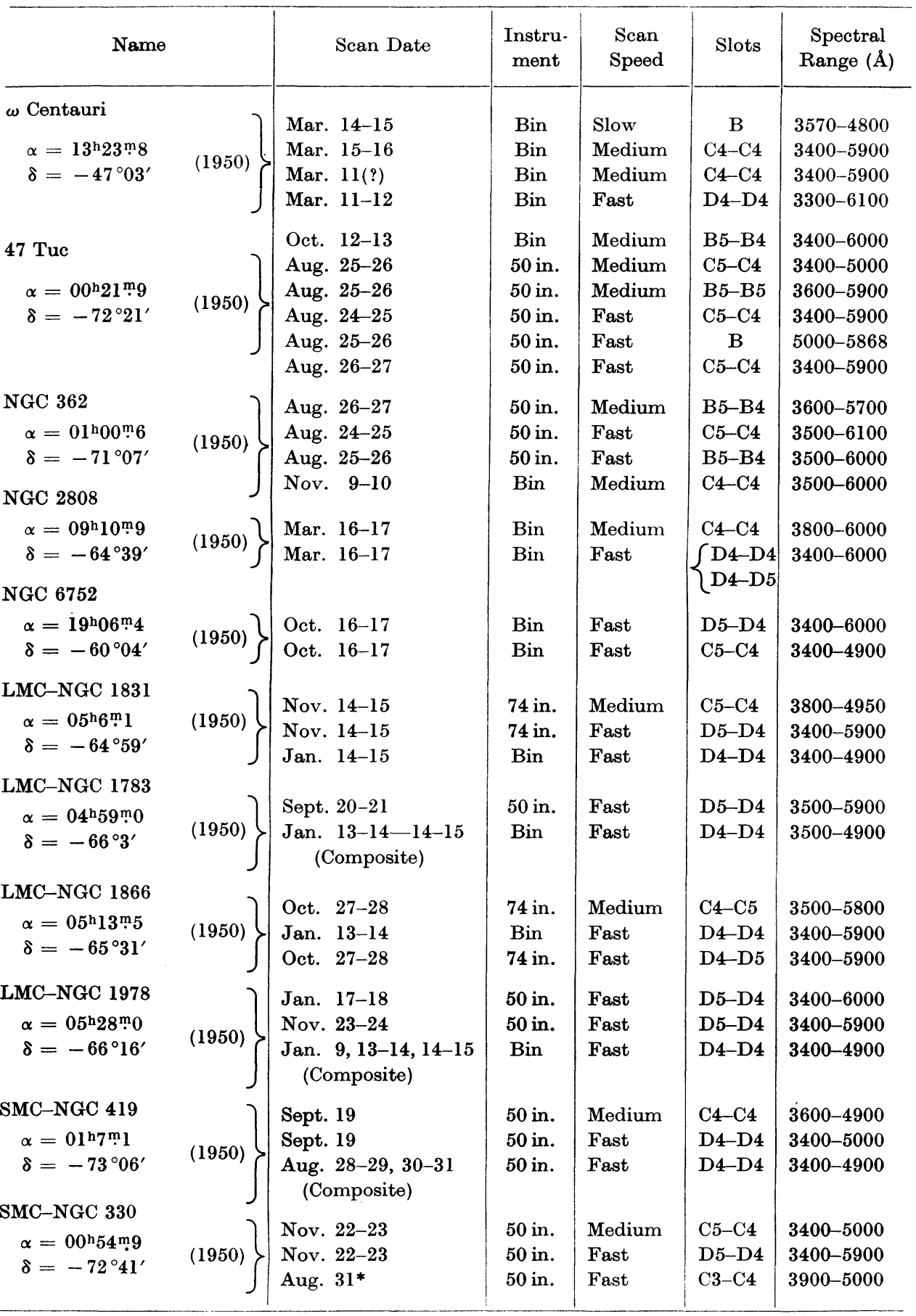

* Very low weight; scale uncertain; sky uncertain. 

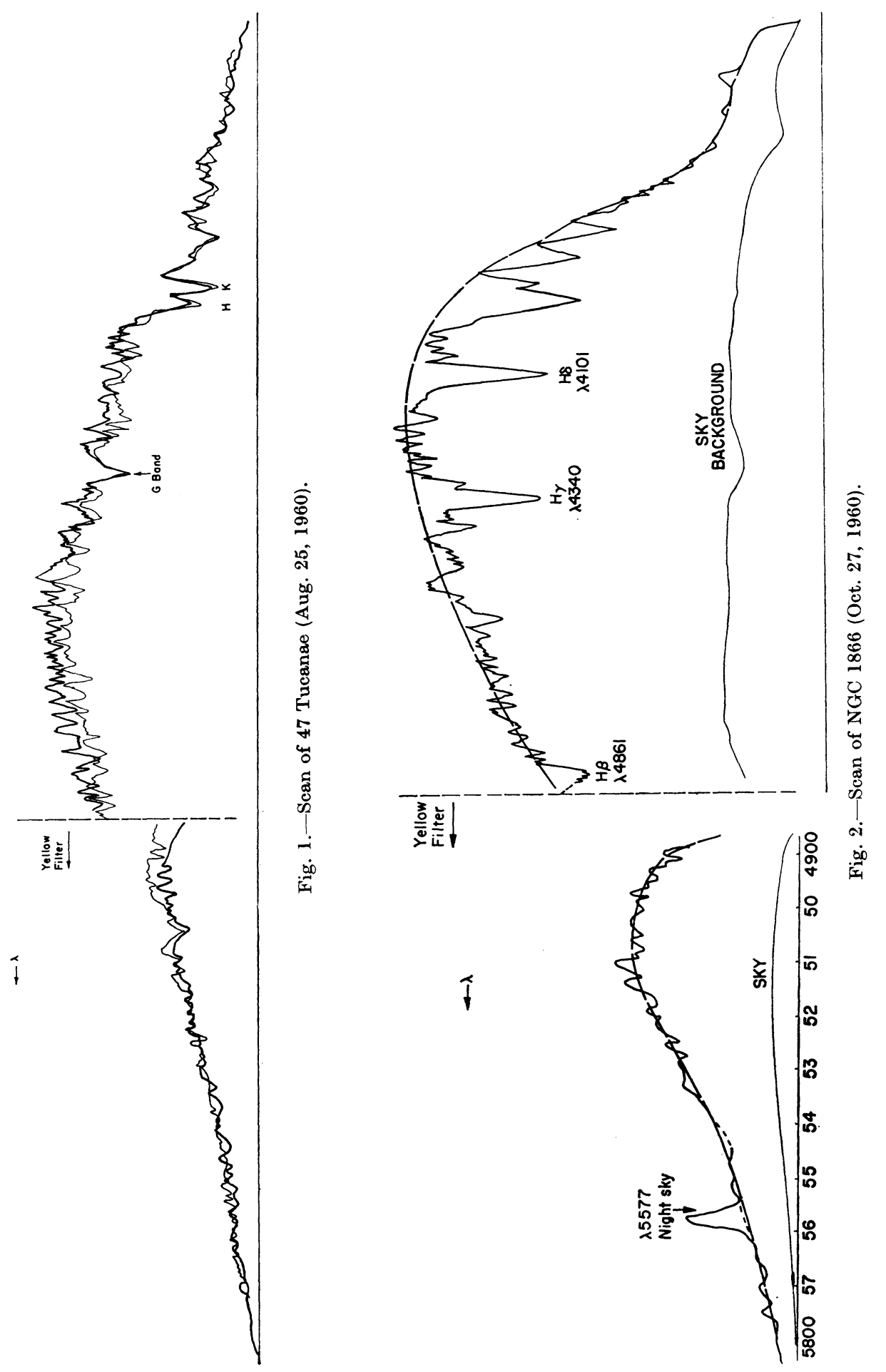
The final results are summarized in Table 2 and in Figures 3, 4, and 5. We measured the scans at the wavelengths chosen by Code (1960) and also at many other positions of the spectrum, particularly in the neighbourhood of the G band ( $\lambda \sim 4308$ ), $\lambda 4227$, the " $H$ " and " $K$ " lines $\lambda 3968$ and 3933 , and also numerous points in the ultraviolet. We compared the clusters individually with stars observed by Code.

TABLE 2

GLOBULAR ClUSter MAGNitUdes RELATIVE to $1 / \lambda_{0}=1 \cdot 80$

\begin{tabular}{|c|c|c|c|c|c|c|c|c|c|}
\hline \multicolumn{2}{|c|}{$1 / \lambda^{*}=2 \cdot 94$} & $2 \cdot 74$ & $2 \cdot 59$ & $2 \cdot 48$ & $2 \cdot 39$ & $2 \cdot 18$ & $1 \cdot 975$ & $1 \cdot 80$ & $1 \cdot 72$ \\
\hline$\omega$ Centauri & $2 \cdot 12$ & $1 \cdot 56$ & $1 \cdot 19$ & $0 \cdot 83$ & $0 \cdot 79$ & $0 \cdot 43$ & $0 \cdot 21$ & $0 \cdot 0$ & -0.25 \\
\hline Est. error & $0 \cdot 40$ & $0 \cdot 10$ & $0 \cdot 10$ & $0 \cdot 05$ & $0 \cdot 05$ & $0 \cdot 10$ & $0 \cdot 10$ & $0 \cdot 10$ & $0 \cdot 40$ \\
\hline 47 Tuc & $2 \cdot 25$ & $1 \cdot 90$ & $1 \cdot 75$ & $1 \cdot 15$ & $1 \cdot 09$ & 0.53 & $0 \cdot 22$ & 0.0 & $-0 \cdot 10$ \\
\hline Est. error & $0 \cdot 25$ & $0 \cdot 15$ & $0 \cdot 08$ & $0 \cdot 05$ & $0 \cdot 05$ & $0 \cdot 04$ & $0 \cdot 05$ & $0 \cdot 12$ & $0 \cdot 15$ \\
\hline NGC 362 & $1 \cdot 88$ & $1 \cdot 65$ & $1 \cdot 34$ & $1 \cdot 03$ & $0 \cdot 86$ & $0 \cdot 48$ & $0 \cdot 28$ & $0 \cdot 0$ & $-0 \cdot 15$ \\
\hline Est. error & 0.50 & $0 \cdot 50$ & $0 \cdot 20$ & $0 \cdot 10$ & $0 \cdot 05$ & $0 \cdot 04$ & $0 \cdot 10$ & $0 \cdot 15$ & $0 \cdot 25$ \\
\hline NGC 2808 & $2 \cdot 18$ & $1 \cdot 96$ & $1 \cdot 63$ & $1 \cdot 33$ & $1 \cdot 11$ & $0 \cdot 71$ & $0 \cdot 38$ & $0 \cdot 0$ & $-0 \cdot 10$ \\
\hline Est. error & & $0 \cdot 07$ & $0 \cdot 10$ & $0 \cdot 15$ & $0 \cdot 05$ & $0 \cdot 08$ & $0 \cdot 15$ & $0 \cdot 20$ & $0 \cdot 30$ \\
\hline LMC-NGC 1831 & $2 \cdot 17$ & $1 \cdot 78$ & $0 \cdot 97$ & $0 \cdot 49$ & $0 \cdot 46$ & $0 \cdot 26$ & $0 \cdot 14$ & $0 \cdot 0$ & $-0 \cdot 06$ \\
\hline Est. error & & $0 \cdot 15$ & $0 \cdot 07$ & $0 \cdot 05$ & $0 \cdot 10$ & $0 \cdot 05$ & $0 \cdot 07$ & & \\
\hline LMC-NGC 1866 & $1 \cdot 75$ & $1 \cdot 21$ & $0 \cdot 40$ & $0 \cdot 15$ & $0 \cdot 13$ & $0 \cdot 07$ & $-0 \cdot 12$ & $0 \cdot 0$ & $0 \cdot 18$ \\
\hline Est. error & $0 \cdot 40$ & $0 \cdot 20$ & $0 \cdot 05$ & $0 \cdot 07$ & $0 \cdot 05$ & $0 \cdot 10$ & $0 \cdot 20$ & $0 \cdot 15$ & $0 \cdot 25$ \\
\hline LMC-NGC 1783 & $2 \cdot 41$ & $1 \cdot 80$ & $1 \cdot 26$ & $0 \cdot 76$ & $0 \cdot 54$ & $0 \cdot 46$ & $0 \cdot 26$ & $0 \cdot 0$ & $-0 \cdot 07$ \\
\hline Est. error & $0 \cdot 80$ & $0 \cdot 20$ & $0 \cdot 15$ & $0 \cdot 10$ & $0 \cdot 10$ & $0 \cdot 10$ & $0 \cdot 10$ & & \\
\hline NGC 6752 & $1 \cdot 92$ & $1 \cdot 46$ & $1 \cdot 21$ & $0 \cdot 88$ & $0 \cdot 78$ & $0 \cdot 46$ & $0 \cdot 22$ & $0 \cdot 0$ & -0.06 \\
\hline Est. error & $0 \cdot 40$ & $0 \cdot 15$ & $0 \cdot 10$ & $0 \cdot 10$ & $0 \cdot 05$ & $0 \cdot 05$ & $0 \cdot 10$ & $0 \cdot 05$ & $0 \cdot 10$ \\
\hline LMC-NGC 1978 & $2 \cdot 04$ & $1 \cdot 63$ & $1 \cdot 33$ & $0 \cdot 99$ & $0 \cdot 78$ & $0 \cdot 47$ & $0 \cdot 25$ & $0 \cdot 0$ & $-0 \cdot 10$ \\
\hline Est. error & $0 \cdot 20$ & $0 \cdot 20$ & $0 \cdot 10$ & $0 \cdot 20$ & $0 \cdot 10$ & $0 \cdot 10$ & $0 \cdot 15$ & $0 \cdot 10$ & $0 \cdot 13$ \\
\hline SMC-NGC 330 & $0 \cdot 34$ & $0 \cdot 28$ & $-0 \cdot 13$ & $-0 \cdot 11$ & $-0 \cdot 10$ & -0.07 & $-0 \cdot 03$ & $0 \cdot 0$ & $0 \cdot 16$ \\
\hline Est. error & $0 \cdot 20$ & $0 \cdot 05$ & $0 \cdot 10$ & $0 \cdot 07$ & $0 \cdot 05$ & $0 \cdot 05$ & $0 \cdot 10$ & $0 \cdot 15$ & $0 \cdot 20$ \\
\hline
\end{tabular}

$* 1 / \lambda$ is given in reciprocal microns.

The primary entries in Table 2 give the brightness $F(1 / \lambda)$ of the globular cluster in magnitude relative to the brightness at $1 / \lambda_{0}=1 \cdot 80$. One magnitude corresponds to $4 \mathrm{~dB}$. The secondary entries for each cluster give a liberal estimate of the error involved. The size of the error increases at the limits of the scan because of the decreased sensitivity of the photocell, and in the ultraviolet because of the increased uncertainty in the atmospheric transmission. 
In Figures 3, 4, and 5 the brightness is plotted in magnitudes against $1 / \lambda$ (in reciprocal microns as abscissa). Vertical dotted lines indicate the spread of the

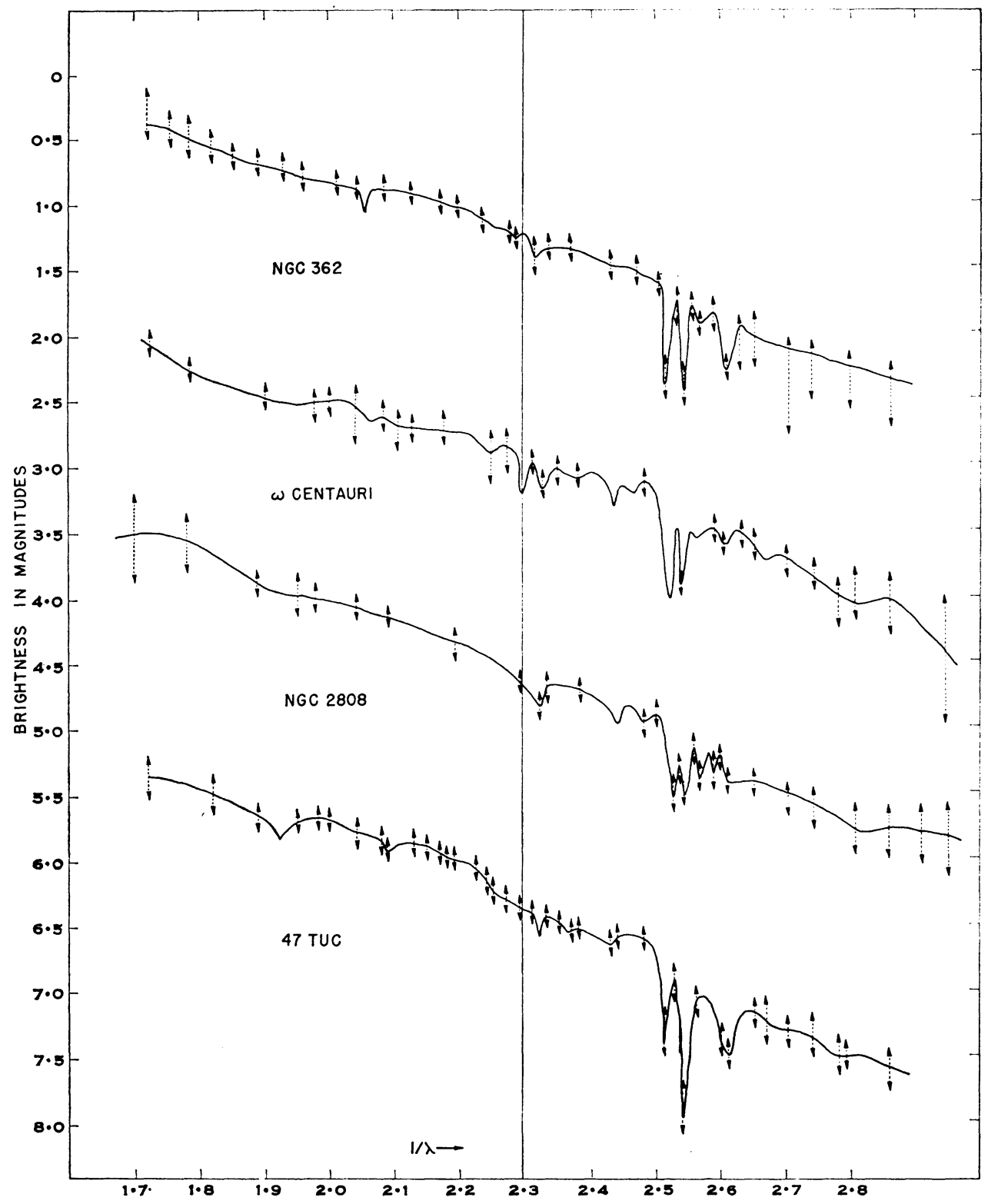

Fig. 3.-Energy distributions for NGC 362, $\omega$ Centauri, NGC 2808, and 47 Tucanae.

individual spectrum scans. They depict liberally estimated uncertainties, not errors calculated by statistical theory.

We deemed it best to retain the "wiggles" in the individual curves. Some of them are almost certainly spurious, but others, such as those corresponding to the 
"G-band" and particularly to the $\mathrm{H}$ and $\mathrm{K}$ lines, seem to be unambiguously established. We must emphasize, however, that these fine features of the spectra can be observed only with slit spectrograms; the resolution of the scanner is too low and the

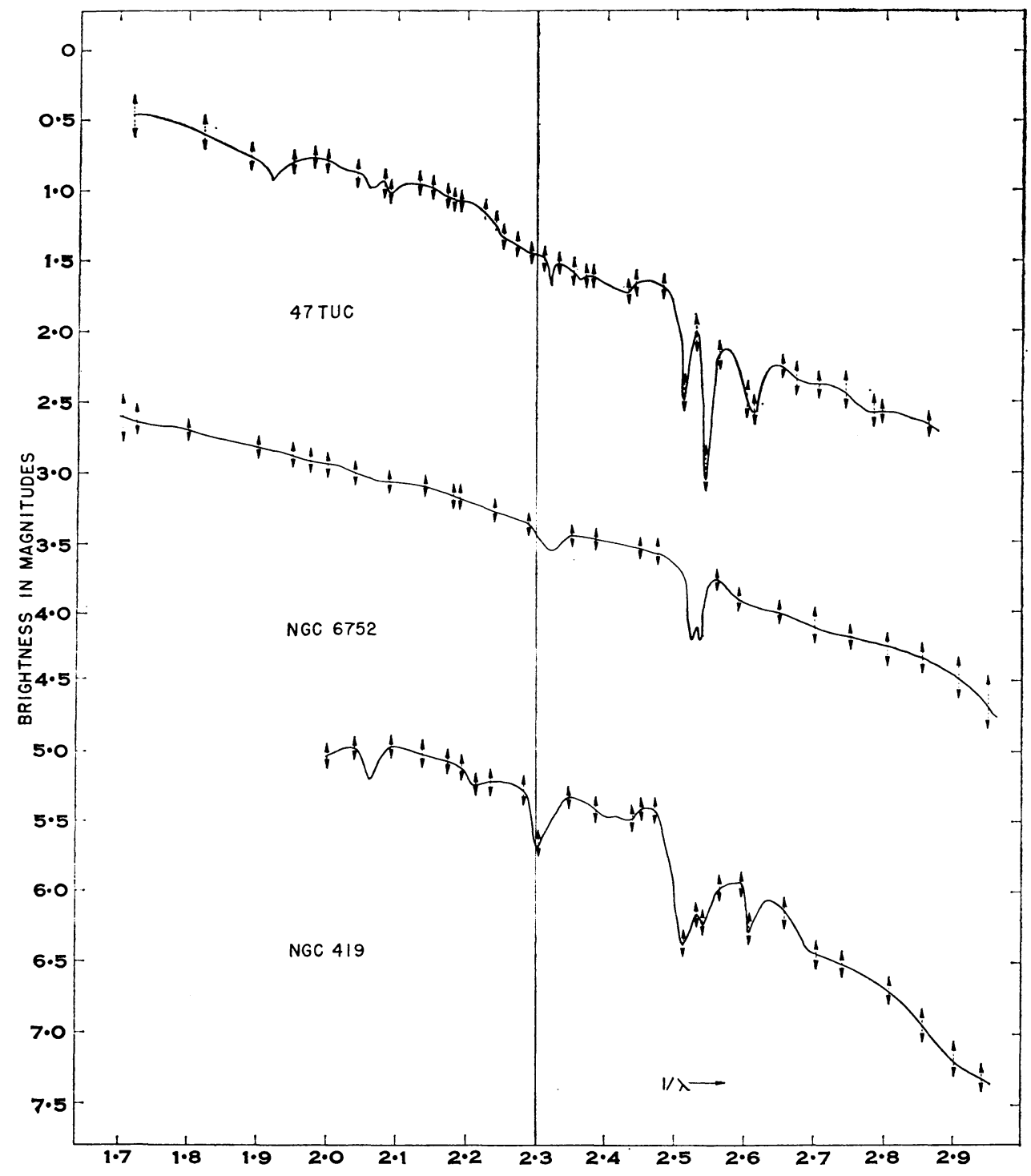

Fig. 4.-Energy distributions for 47 Tucanae, NGC 6752, and NGC 419.

noise level too high to permit delineation of many spectral characteristics that are useful for assessing absolute magnitude effects, metal deficiencies, etc.

In Figure 3 we compare NGC 362, $\omega$ Centauri, NGC 2808, and 47 Tucanae. Of these clusters, 47 Tucanae is probably the most "metal rich". There is a sharp drop in the continuum in the neighbourhood of the $H$ and $K$ lines because of the 
many overlapping metallic lines in this spectral region. The spectral distribution fits $\eta$ Hercules G4 III for $1 / \lambda<2 \cdot 6$, but is brighter than this star in the ultraviolet $(1 / \lambda>2 \cdot 6)$ by about $0 \cdot 3 \mathrm{mag}$. If we fit the energy distribution of 47 Tucanae to

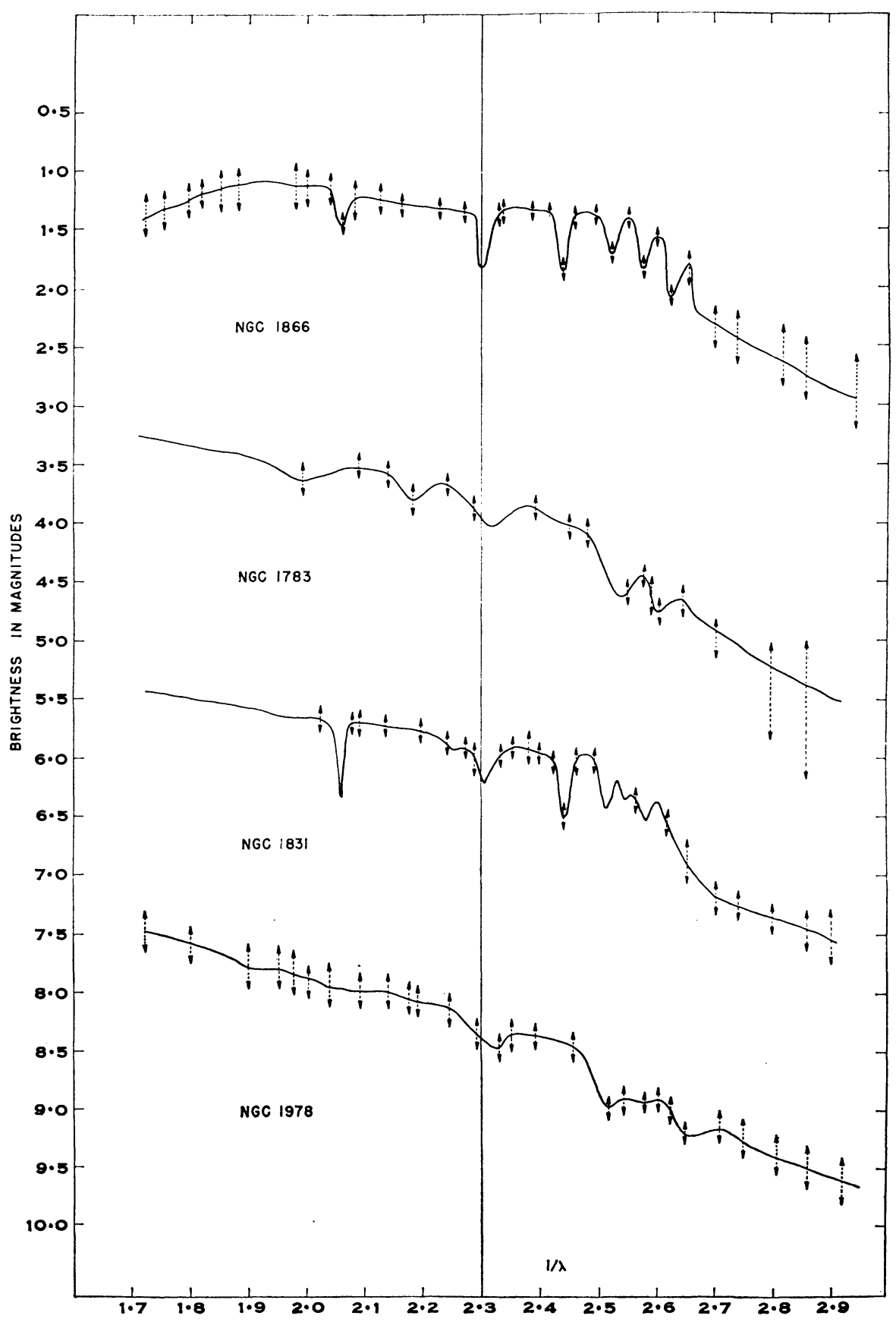

Fig. 5.-Energy distributions for NGC 1866, NGC 1783, NGC 1831, and NGC 1978. 
that of 31 Comae G0 III, we find that a good fit can be obtained for $1 / \lambda>2 \cdot 3$. That is, 47 Tucanae is redder than G0 III. It probably corresponds to about G2 III.

The cluster NGC 2808 appears to have about the same energy distribution as 47 Tucanae; i.e. it corresponds to about G2 III, being redder than a G4-dwarf. It appears to be more metal deficient than 47 Tucanae but is certainly not an extreme type II population object such as a subdwarf.

The energy distribution in $\omega$ Centauri falls between that of a G4 V star such as 51 Pegasi, and that of a G2 V star such as 16 Cyg A. It appears to be metal deficient in comparison with 47 Tucanae.

We experienced some difficulty in measuring the ultraviolet energy distribution in NGC $362(1 / \lambda>2 \cdot 7)$ when the highest resolution $24 \AA$ was sought, so we have disregarded these measurements in drawing the final curve. The cluster appears to be definitely metal deficient; its energy distribution fits that of a $\mathrm{G} 4 \mathrm{~V}$ star to within the estimated error of the measurements, although it may be slightly redder. It is much bluer than a G4 III star. We estimate its energy distribution as corresponding to that of a G5 V star.

In Figure 4 we compare 47 Tucanae with NGC 6752 and NGC 419, a globular cluster in the Small Magellanic Cloud. NGC 6752 fits 51 Pegasi G4 V over nearly the entire range covered. Because of its faintness, NGC 419 was observed only in the blue. In the interval $2 \cdot 0<1 / \lambda<2 \cdot 6$ its energy distribution fits that of 51 Pegasi G4 V but it has strong $H$ and $K$ lines and a strong $G$ band. The weakness of the cluster in the ultraviolet may partly arise from observational error, but part of it may arise from the influence of the extremely red stars noted by Arp (1958). Further observations of this cluster are essential, since the present fragmentary data would suggest that the cluster is abnormally rich in metals.

Figure 5 shows the data for the clusters in the Large Magellanic Cloud. NGC 1866 and NGC 1831 were observed with higher resolution, i.e. $44 \AA$, than the other two clusters, NGC 1783 and NGC 1978, which could be measured only with a resolution of $106 \AA$. The energy distribution in NGC 1866 resembles that of a late A-type star. In the interval $1 \cdot 8<1 / \lambda<2 \cdot 6$ the slope of the energy curve corresponds to a spectral class a little later than A5 III, although the curve shows a drop in the extreme red. The Balmer jump corresponds to that of $\beta$ Tri spectral class A5 III. The strong Balmer lines are easily visible upon the tracing and show clearly that the spectral class is definitely $A$, in agreement with Thackeray's result.

The energy distribution in the cluster NGC 1831, which was also observed with the 74-inch reflector, shows a good fit to 110 Herculi's F6 V in the interval $1 \cdot 7<1 / \lambda<2 \cdot 5$, but the Balmer jump seems to be too large and the ultraviolet portion of the spectrum appears to be relatively too faint by about 0.5 mag in the region $2 \cdot 65<1 / \lambda<2 \cdot 9$. Here again we must emphasize that the ultraviolet data are uncertain and that further observations should be obtained. The Balmer lines still seem to be relatively strong.

The clusters NGC 1783 and NGC 1978 are fainter than NGC 1866 and NGC 1831. Unfortunately we were unable to observe them with the 74-inch telescope. Not only is the spectral purity lower but the influence of sky background is greater, particularly in the ultraviolet. The energy distribution in NGC 1783 appears to 
resemble that of 51 Pegasi G4 V for $1 / \lambda<2 \cdot 65$. In the ultraviolet the errors appear to be large because of effects of the sky. The star is bluer than $\eta$ Hercules G4 III except in the ultraviolet. The energy distribution in NGC 1978 seems to fit that of a G2 V star to within the observational error.

Note that what we have derived are energy distribution equivalents, not spectral classes. The results are summarized in Table 3. Among globular clusters associated with our Galactic System, the spectral classes tend to be earlier than the energy distribution equivalents-which is exactly the effect we would anticipate for stars that were deficient in metals! The exception is 47 Tucanae, which everyone agrees has a normal or nearly normal metal/hydrogen ratio. Because accurate spectral classes are not now available for the clusters in the Magellanic Clouds, similar comparisons cannot be made. The fragmentary data do suggest that perhaps the metal/hydrogen ratio tends to be more nearly "normal" in the globular clusters in the Clouds than in those of our own Galaxy.

TABLE 3

ENERGY DISTRIBUTION EQUIVALENTS FOR GLOBULAR CLUSTERS

\begin{tabular}{|c|c|c|c|}
\hline Name & NGC & $\begin{array}{c}\text { Energy Distribution } \\
\text { Equivalent }\end{array}$ & Spectral Class \\
\hline \multirow[t]{3}{*}{47 Tucanae } & 104 & G2 III & G3 \\
\hline & 362 & G4 V & F8 \\
\hline & 2808 & G2 III & F7 \\
\hline \multirow[t]{2}{*}{$\omega$ Centauri } & 5139 & G4 V & F8 \\
\hline & 6752 & G4 V & F6 \\
\hline SMC Cluster & 419 & G4 V & G \\
\hline \multirow[t]{4}{*}{ LMC Clusters } & 1783 & G4 V & F5 \\
\hline & 1831 & F6 V & G5 \\
\hline & 1866 & A8 III & $\mathbf{A}$ \\
\hline & 1978 & G2 V & $G$ \\
\hline
\end{tabular}

Note that the energy distribution equivalent of NGC 1783 is later than the assigned spectral class, whereas the opposite is true for NGC 1831. Possibly the spectral classes are themselves in error; the energy distributions in NGC 419, NGC 1978, and NGC 1783 are very similar, as are their colour-magnitude arrays.

No emphasis can be laid on the amount of the quantitative difference between the energy distribution equivalents and the spectral classes. In each instance one deals with the composite spectra of a host of stars. A stellar distribution ranging from F0 to K5 may give a spectral class resembling G2, but the energy distribution may differ from that of a G2 star.

The present investigation suggests that spectral scans of star clusters may be helpful in getting an estimate of the metal/hydrogen ratio. Before the data can be interpreted quantitatively, it will be necessary to supplement it with spectroscopic observations (for line intensities), colour-magnitude arrays, and luminosity functions. 
There is some question whether spectral scans of the type we have obtained are the most useful kind of observations to be secured with a spectral scanner. When quantitative studies of the composite spectra of clusters are carried out, it will be possible to select certain spectral features for special attention. An extension of the Strömgren photometric technique to globular clusters is clearly suggested, but the actual spectral intervals must be chosen with care. Furthermore, attention must be paid to getting spectral energy distributions for a variety of stars of different hydrogen/metal ratios and surface temperatures.

We secured spectral scans of NGC 330 in the SMC. This object is an open cluster containing many stars of early spectral type; Figure 6 shows its energy distribution. Although the Balmer jump corresponds to that of a B3 star, the energy distribution and the strength of the hydrogen lines correspond to a later spectral class.

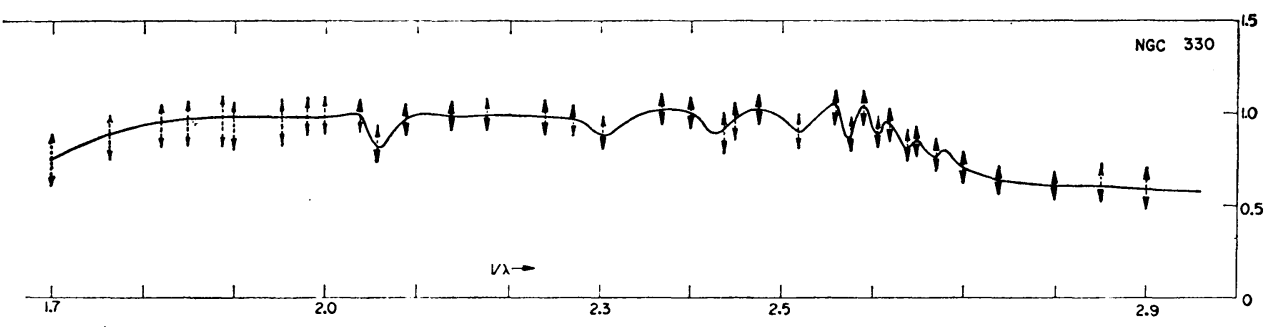

Fig. 6.-Energy distribution for NGC 330 .

Very recently, van den Bergh and R. C. Henry (1962) have published results of their spectrophotometric studies of 21 globular clusters with an effective resolution of about $35 \AA$. Many of their objects were highly reddened, but they found it possible to segregate effects of interstellar reddening from those of intrinsic colour differences. They also noted a discontinuity in spectral energy distribution near $\lambda 4000$ to be correlated with metallic line strength in globular clusters and with metal/hydrogen ratio in main sequence stars. They tabulate $m(1 / \lambda)$ at intervals of $100 \AA$ from $\lambda 3400$ to $\lambda 5200$.

The Krons have measured a number of southern globular clusters in six colours. Their measurements are in good agreement with ours, over the wavelength regions which we have covered.

Participation in this program by one of us (LHA) was made possible by the award of a senior post-doctoral National Science Foundation Fellowship and generous cooperation from the Australian National University, while the other (DJF) was supported by a postgraduate studentship of the CSIRO. Analysis of the observations was carried out as part of a project sponsored by the United States Air Force under Contract No. AF 49(638)-807 with the University of Michigan and monitored by the Air Force Office of Scientific Research of the Air Research and Development Command.

Mr. Herbert Rood of University of Michigan Observatory checked most of the reductions and compiled a summary of the published data. 


\section{References}

ARP, H. C. (1958.-A.J. $63: 273-82$.

van Den Bergh, S., and Henry, R. C. (1962).-Publ. David Dunlap Obs., Toronto 2: 218-313. Code, A. (1960).- "Stars and Stellar Systems." (General Ed. G. P. Kuiper.) Vol. VI. "Stellar Atmospheres." (Ed. J. L. Greenstein.) pp. 50-87. (Univ. Chicago Press.)

LILLER, W. (1957).-P.A.S.P. 69 : 511-17.

\section{Discussion}

Feast: Could Dr. Aller say where the spectral types he quoted come from? Are they unpublished data?

Aller : We collected them from the available literature. Of course we would like better classifications.

Eggen: The problem of accurate classification of weak-lined stars must be kept in mind.

Aller: The analysis of such a composite spectrum is very difficult in practice. One must select stars of different metal/hydrogen ratios and use the observed colour-magnitude arrays to construct a synthetic composite spectrum and energy distribution. Further, one must have a good description of the composite spectrum, obtained with as high resolution as practicable.

Rodgers: I agree with Dr. Aller that in the interpretation of the integrated scans of clusters the Hess diagram is most important. Differentiation between the intermediate-age clusters and halo clusters of intermediate abundances seems difficult.

Aller: We cannot interpret energy scans correctly without other data, such as adequate colour-magnitude diagrams. But when we have a colour-magnitude diagram with the correct number of stars in each interval of magnitude and colour, spectrograms from which line intensities may be measured, and energy scans, we should be able to get a result for the ratio of hydrogen to metals.

Heard: van den Bergh and Henry have studied 24 globular clusters visible in northern latitudes by means of spectrum scans, and have found several discriminants which correlate well with the Morgan line-strength classifications and serve to separate the clusters as to metal abundance.

Aller: The method must of course be calibrated; van den Bergh and Henry have suggested some leads that may be applied profitably to Magellanic Cloud clusters, when better spectral classifications are available.

As far as our cluster data suggest, as yet there is no evidence for a different metal/hydrogen ratio in the Magellanic Clouds than in the Galaxy.

\section{SHORT-PERIOD VARIABLES IN THE MAGELLANIC CLOUDS}

\section{A. D. Thackeray \\ Radcliffe Observatory}

Our knowledge of short-period variables in the Magellanic Clouds remains rather meagre despite the considerable effort that has been put into discovering them. Original failures to detect RR Lyrae variables at-the expected magnitude of 17.5 led to a temporary belief that perhaps the Clouds contained virtually no Population II. However, the Radcliffe discoveries of RR Lyrae variables in the globular clusters NGC $121(3), 1466(44), 1978(2)$, and $2257(17+8)$, put the existence of a Population II component in both Clouds beyond doubt. Moreover, the published light curves and periods of variables in NGC 121 (Thackeray 1958) and NGC 2257 